\title{
Paper, Pen and Touch
}

\author{
Moira C. Norrie \\ Institute for Information Systems, ETH Zurich \\ CH-8092 Zurich, Switzerland \\ norrie@inf.ethz.ch
}

\begin{abstract}
It has long been recognised by researchers that the affordances of paper are likely to ensure that it will continue to be in widespread use in the work place, homes and public spaces. Consequently, numerous research projects have investigated ways of integrating paper with digital media and services. In recent years, a lot of this research has revolved around the digital pen and paper technology developed by the Swedish company Anoto, since it offers a robust solution for tracking the position of a pen on paper. While the commercial sector has tended to focus on applications related to the capture of handwriting, many of these research projects have investigated the use of the pen for real-time interaction and possibilities of turning paper into an interactive medium.

Researchers were also quick to realise that digital pen and paper technology could be adapted to support other forms of pen-based interaction and have developed digital whiteboards and tabletops based on the technology. In addition, some systems have combined the technology with touch devices to support bimanual pen and touch interfaces. In the case of document manipulation, this means that touch could be used to perform actions such as a moving a document or turning pages, while the pen could be used to select elements within a document or to annotate it. Further, there are projects which have integrated the work on interactive paper and pen-based interaction on digital tabletops, investigating ways of allowing users to transfer document elements back and forth between paper and digital surfaces.

Despite the success of these research projects in terms of demonstrating the capabilities of digital pen and paper technology and how it could be exploited to support a wide variety of everyday tasks, there are still some technical and non-technical issues that need to addressed if there are to be major breakthroughs in terms of widespread adoption. The first part of the talk will review research in the field, while the second part will examine these issues and the way ahead.
\end{abstract}

